\title{
Study of Energy Efficiency Characteristics of a Hydraulic System Compo- nent
}

\section{Dr. Alamgir A. Choudhury, Western Michigan University}

Alamgir A. Choudhury is an Associate Professor of Engineering Design, Manufacturing and Management Systems at Western Michigan University, Kalamazoo, Michigan. His MS and PhD are in mechanical engineering from NMSU (Las Cruces) and BS in mechanical engineering from BUET (Dhaka). His interest includes computer applications in curriculum, MCAE, mechanics, fluid power, and instrumentation $\&$ control. He is a Registered Professional Engineer in the State of Ohio and affiliated with ASME, ASEE, SME and TAP.

\section{Prajna Paramita, Western Michigan University \\ Dr. Jorge Rodriguez P.E., Western Michigan University}

Faculty member in the Department of Engineering Design, Manufacturing, and Management Systems (EDMMS) at Western Michigan University's (WMU). Co-Director of the Center for Integrated Design (CID), and currently the college representative to the President's University-wide Sustainability Committee at WMU. Received his Ph.D. in Mechanical Engineering-Design from University of WisconsinMadison and received an MBA from Rutgers University. His B.S. degree was in Mechanical and Electrical Engineering at Monterrey Tech (ITESM-Monterrey Campus). Teaches courses in CAD/CAE, Mechanical Design, Finite Element Method and Optimization. His interest are in the area of product development, topology optimization, additive manufacturing, sustainable design, and biomechanics. 


\section{Study of energy efficiency characteristics of a hydraulic pump}

\section{Introduction}

Over $80 \%$ of the energy used worldwide comes from finite nonrenewable sources, something that is supposed to increase significantly in coming years. According to the U.S. Energy Information Administration's International Energy Outlook 2017, the world's energy consumption is expected to increase by $48 \%$ between the years 2015 and 2040 . Recognizing the global demand for energy and its impact on the environment, practice of energy efficiency in all products and processes is essential for future sustainable industries.

A fluid power system [1] transforms available forms of energy to mechanical energy through the use of fluid media. It also deals with the transmission and control of forces and motion using a pressurized fluid. In general, a fluid power system, specifically the hydraulics system, is a cost effective solution to problems requiring high power density and large reversible forces or torque in an application. It is also easy to control and can be integrated with a mechanical or electrical system in complex applications. As pressurized fluid circulates, hydraulic systems waste much of their energy, regardless of the amount of work output. Most industrial hydraulic applications are not designed with the goal of minimizing their energy consumption [2], therefore thermodynamically, they tend to be inefficient. Various studies [3, 4] investigated methods to incorporate the overall efficiency of a hydraulic system in the design of applications.

In typical hydraulic systems, overall efficiency may vary from $6 \%$ to $60 \%$. Literature on energy conservation and efficiency research includes a variety of efforts to analyze hydraulic systems under different types of conditions. Borghi et al. [5] investigated the problem of reducing power dissipation of hydraulic circuits in off-road vehicles. To conserve energy, it used a lumped parameter approach for an alternative hydraulic circuit architecture of agricultural tractors. Kilic et al. [6] presented a study to predict pressures in the cylinder chambers of a variable-speed pump that was monitored and controlled through the use of a neural network with linear and Kalman filters. Another work [7] by Puddu and Paderi proved that the behavior of real gas has a considerable influence on the size of accumulators used for kinetic energy recovery of vehicles. Thermodynamic properties of gases were analyzed to find the differences between ideal and real gas compressibility. Different gases were tested as charging fluid in a hydro-pneumatic accumulator. The results showed distinctive characteristics in volumetric gas properties and thermal efficiency of the accumulator. Sola et al. [8] proposed to improve energy efficiency in an industrial motor system using a multicriteria analysis. It recommended the dissemination of multicriteria decision aiding to support the decision makers in industries and to improve energy efficiency in systems powered by electric motors. Ramakrishnan et al. [9] developed a multiobjective design optimization procedure based on an evolutionary strategy algorithm that maximizes the energy efficiency of a hydraulic system. The study showed that optimizing the system parameters increased the energy efficiency by $3 \%$ and hydraulic regeneration efficiency by $17.3 \%$.

Typically, a hydraulic system is composed of components and a circuit instrumentation, as well as a control system. Connections between all the hoses with respective units along with the flow, 
temperature, pressure, and RPM sensors create the hydraulic circuit of the system where pumps and motors transform the power.

In industrial hydraulic systems, high-power pumps are designed for low energy consumption, but the low-power pumps are usually focused on low investment costs sacrificing their energy efficiency. Conventional variable speed hydraulic drives exhibit the ruggedness, weight, and controllability required for a large system; however, they have acceptable efficiency at the full load and efficiency drops as the load changes.

\section{Energy losses in a hydraulic system}

The viscosity of a fluid determines the resistance to flow. With higher viscosity, the fluid friction increases and energy is dissipated as heat. Energy losses occur in various forms when there is a change in the cross-section of the flow path and the direction of the flow in valves, hoses, couplings, filters etc. The more path fluid travels, the greater the losses. The nature of resistance of valves, hoses, and pipes can be determined using experimental methods. A complete analysis of a system requires both analytical and experimental methods.

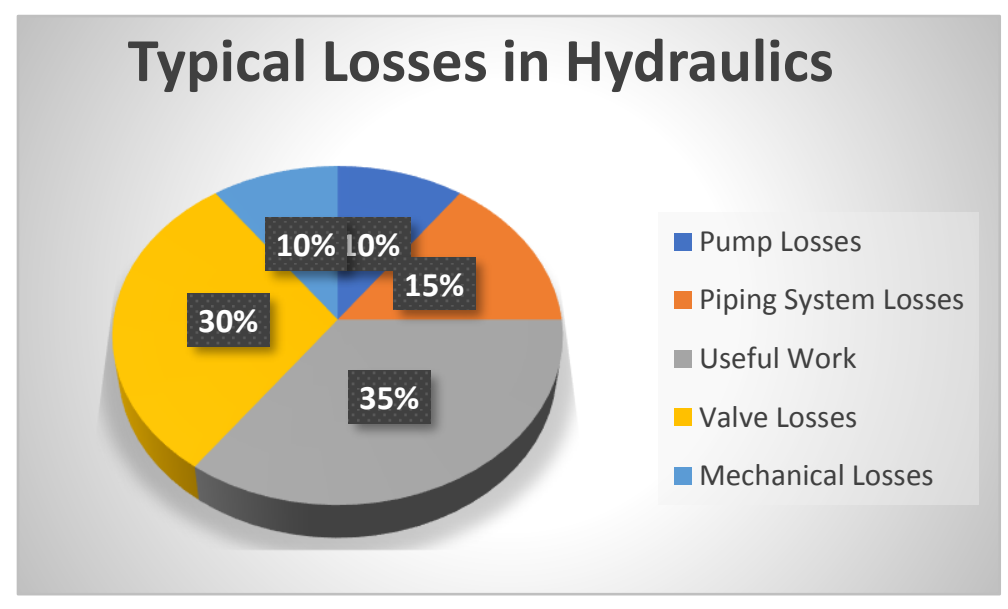

Figure 1: Typical losses in hydraulics with variable pump

Losses in pumps are determined by overall efficiency, which is a product of volumetric efficiency and mechanical efficiency of the system. Losses on hoses and other components are comprised of linear losses and can be approached as local losses with relatively small values. Therefore, it is very important to design a hydraulic system with hoses that have the correct material, shape, and diameter to ensure as little friction as possible. Energy losses in rotary motors are similar to pumps. A typical breakdown of losses in a hydraulic system with a variable displacement pump is shown Figure 1.

The US Department of Energy compiled more than 2.4 million pumps from various industries and studied the total energy consumption of those pumps. Half of the pumps were of $4 \mathrm{~kW}$ or less power. The upper curve in Figure 2 depicts the power consumed by the pumps in each power range. Pumps in the power range from 4 to $15 \mathrm{~kW}$ consume almost $20 \%$ of the energy 
consumed by all pumps in total. Consequently, $50 \%$ of the energy is consumed by pumps with a rated power of $40 \mathrm{~kW}$ or less [10].

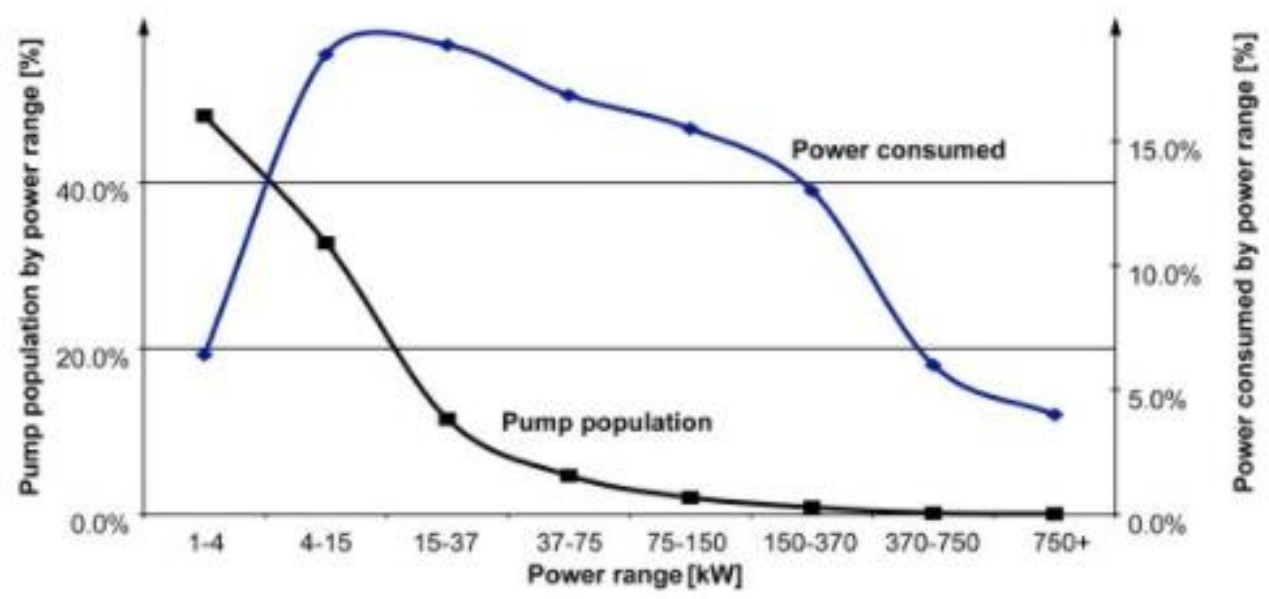

Figure 2: Population of pumps by power range and power consumed in US

This paper presents a study on improving performance and energy efficiency of hydraulic system components, specifically, low power hydraulic pumps. The aim is to develop a hydraulic system to maximize its energy efficiency based on optimal operating conditions. Though the pumps are designed to be most efficient at their rated power, operation in diverse conditions can bring this efficiency to a much lower level. Therefore, control of operating conditions is one area that can be explored to enhance efficiency of a hydraulic system. The idea can be extended to other relevant components as well.

The fluid running through the system is Mobil DTE 24. The pump powering the experiment was Baldor Electric VEM31155 with 2 HP of input power. NI DAQmx module is used to collect all real time data and process those in the LabVIEW environment. To measure the pressure-drop due to friction in two lines, pressure sensors are used while flow is measured using a flowmeter. A hydraulic motor is used to produce the driving torque and rotation of the pump. A torque and RPM sensor is mounted at the pump motors coupling to collect and record input of the torque and shaft RPM.

\section{Prototype development}

The prototype of an application measuring the performance of a small pump is developed in the laboratory using a modular hydraulic system [11]. The power unit of the system consists of an electrically driven pump, reservoir, and supply and return manifolds. The hydraulic system for the study consisted of a small pump and hydraulic motor, flow control valves, relief valves, hoses and fittings. Pressure transducers, flowmeters, thermocouples, and torque/RPM sensor monitor process parameters. Pipes, hoses, and tubes serve as the conductors that distribute fluid between the pump and remaining system components. To perform the fluid control function in a system, three types of valves are used, namely: directional control valves, pressure control valves, and flow control valves. Directional control valves control where in the flow circuit the 
fluid will flow. Pressure control valves limit the maximum pressure and lower pressure in different parts of the system. Flow control valves control the fluid flow rate to evaluate the rate of movement of an actuator in sections of a system. A fluid conditioning system is used to remove contaminants and maintain a proper temperature for hydraulic fluid. As hydraulic oil moves from the reservoir to pump and runs through a system, an additional filter at the return manifold clears impurities prior to its return to the tank. The first system component the oil encounters is the pressure control valve, which sets system pressure. Next comes the directional control valve, which directs the oil to the motor. Oil then moves out into system lines and returns to the reservoir through the directional control valve. Oil from the other side of the piston is returned to the reservoir. A relief valve is located between the pump and the directional control valve to protect the system from potential high-pressure risks. In case of high pressure, some oil will return to the reservoir and ensure pressure does not exit the set limit. A flow control valve restricts the oil flow in ways other than controlling shaft speed and supplies an established volume of oil to the motor.

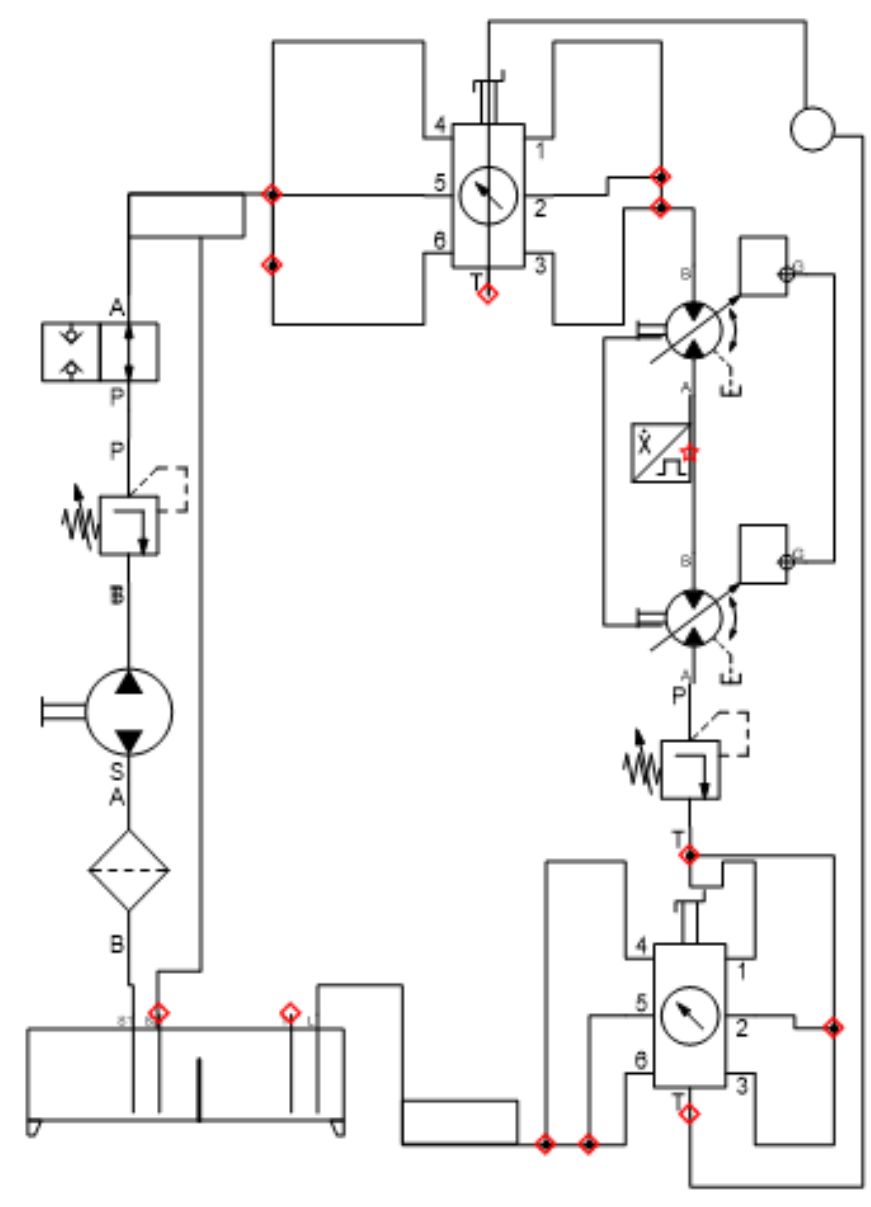

Figure 3: Schematic of the hydraulic system 


\section{NI 9207}
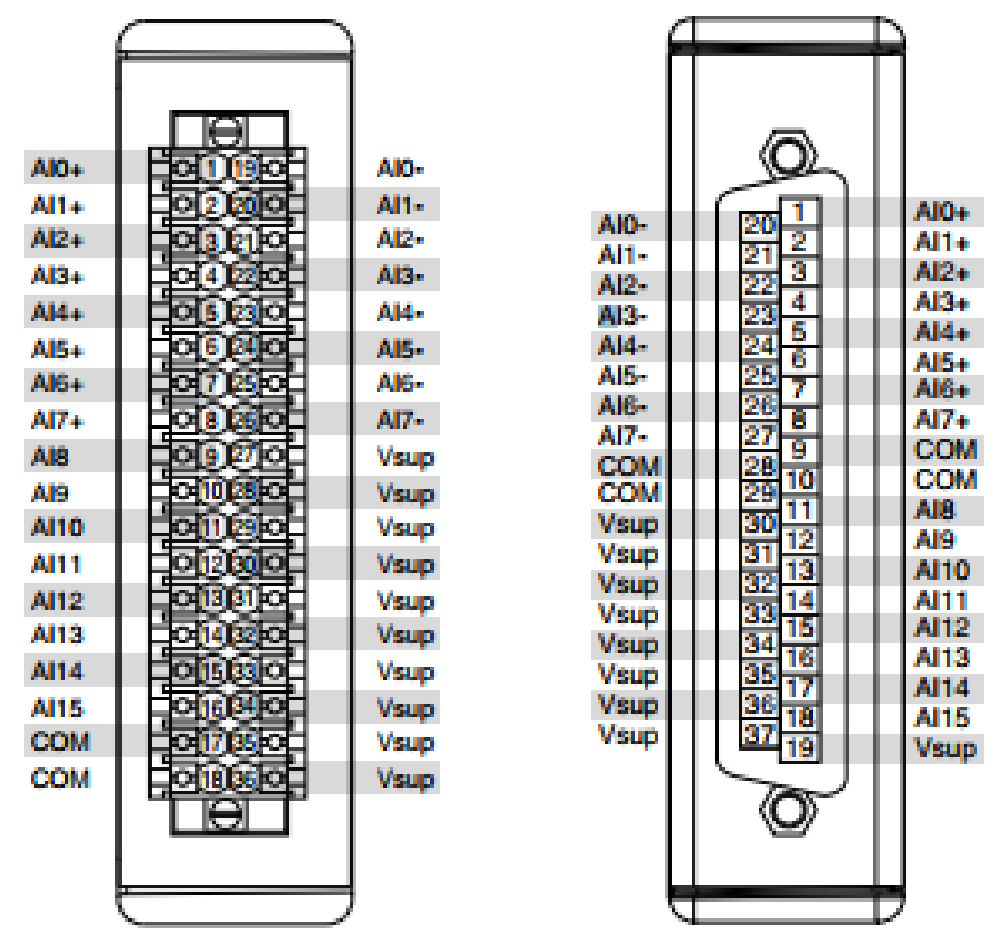

Fig 4: NI 9207 pin out diagram

The pressure, temperature, flow, torque, and RPM measurements are collected using National Instruments DAQ hardware. A cDAQ-9188 chassis is used to collect signals from a NI 9207 analog voltage and current sensing module contained in slot 1. The wiring of the NI 9207 module is shown in Figure 4. The positive lead of power supply is wired directly to a Vsup pin, 19 , with a 2 A quick burn inline fuse and the ground of the power supply is attached to a common ground pin, 10. The positive pressure transmitter lead is connected to a Vsup pin, 30, and the negative lead is connected to a current sensing channel, AI8. The voltage of the cell is measured via the AI0+ and AI0- pins. A $1 \mathrm{M} \Omega$ resistor is connected to pin 28, the common ground and has a common node with the negative voltage lead. The sensors were set to collect data at a frequency of $20 \mathrm{~Hz}$.

Using the graphical programming language of LabVIEW, a Virtual Instrument (VI) was created to collect, display, and record the data from the sensors. The VI Front Panel is seen in Figure 6. In separate charts, real time pressure, temperature, flow, and torque were displayed. The VI allows saving the output in a csv file that is used for detailed analysis. 


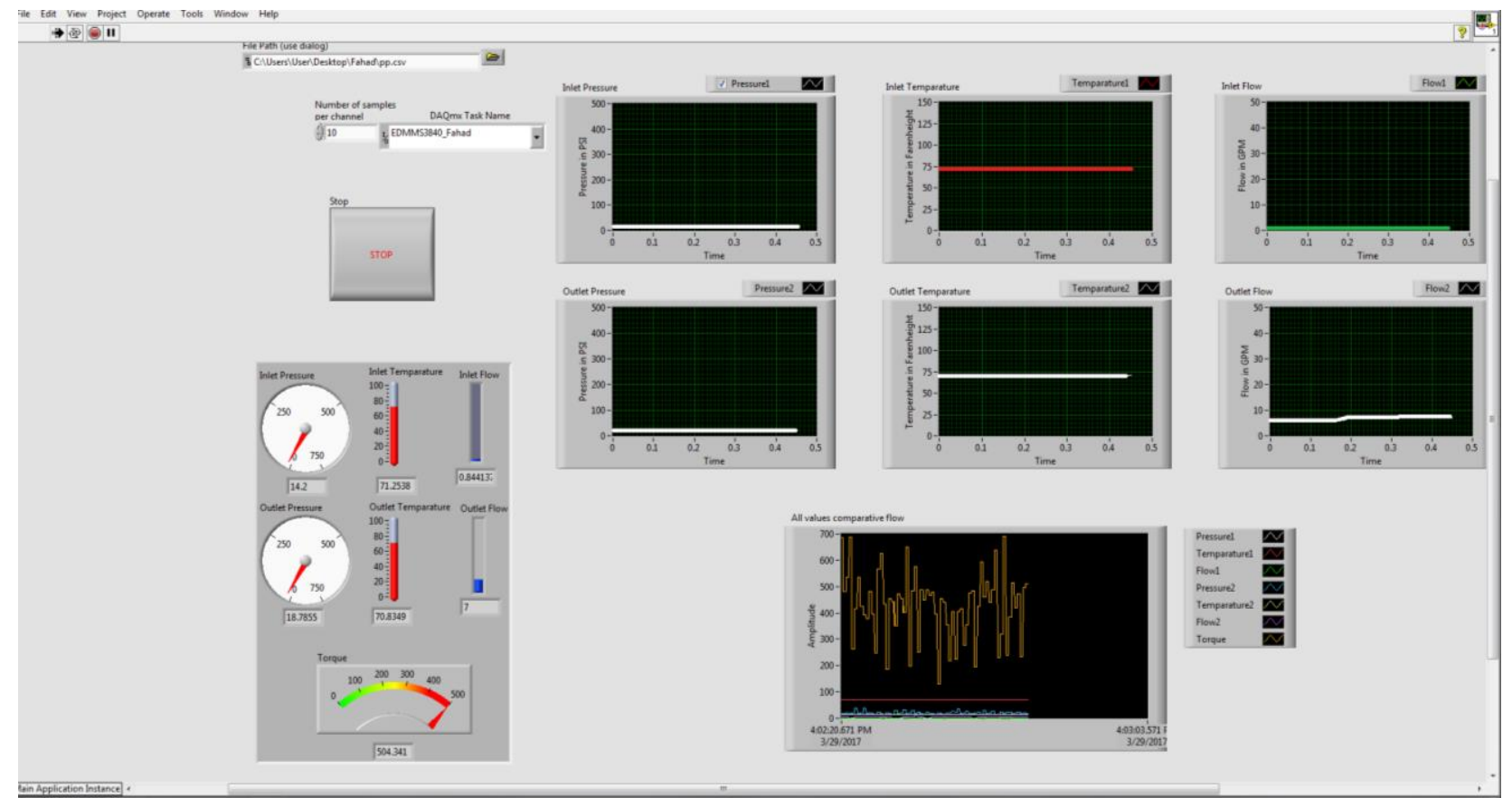

Figure 5: VI front panel

\section{Data Analysis}

Fluid flow characterization in most hydraulic systems can be derived from the Navier Stokes equation

$$
\frac{\partial \rho}{\partial t}+\nabla(\rho V)=0 \ldots
$$

Where, $\rho=$ density, $V=$ flow velocity $\nabla=$ vector divergence operator of a general flow field.

Considering energy input and energy loss in a pump, equation (1) for one dimensional fluid flow simplifies as Bernoulli's equation

$$
\frac{P_{1}}{\gamma}+z_{1}+\frac{V_{1}^{2}}{2 g}+h_{i}-h_{L}=\frac{P_{2}}{\gamma}+z_{2}+\frac{V_{2}^{2}}{2 g} \ldots .
$$

where $h_{i}$ and $h_{L}$ are input energy head and head loss between inlet and exit of a pump.

Utilizing this at pump inlet and exit, the efficiency of the pump can be expressed in terms of output pressure $P$, flow rate $Q$, torque $\tau$ and angular velocity $\omega$ as

$e=\frac{P Q}{\tau \omega}=F_{1}(P, Q, \tau, \omega)$

Again, flow rate $Q$ is a function of pump geometry (displacement volume) $q$, angular velocity $V$, viscosity $\mu$ and temperature $T$. In addition, output pressure $P$ is a function of torque $\tau$, flow rate $Q$, fluid viscosity $\mu$, and temperature $T$.

$Q=F_{2}(q, \omega, \mu, T) \ldots(4)$ 
$P=F_{3}(Q, \tau, \mu, T) \ldots(5)$

Using equation (4) and (5) in (3), the overall efficiency of a pump is represented by the function $e=F(q, \omega, P, Q, \tau, \mu, T) \ldots$

Therefore, the efficiency of a pump in general is a function of a large number of independent variables, most of which can be controlled in a process. The purpose of this study is to optimize the efficiency of a pump by controlling the operational parameters of the process in equation (6). Process data of pump operations under diverse operational conditions are acquired for this purpose. In general, the efficiency function of the pump is complex and nonlinear in nature. Numerical analysis of this type of function can be a cumbersome task. Therefore, the effect of the operational parameters on pump efficiency is established. Sensitivity analysis can be used to investigate the relationship between process parameters of a system, their impacts on the outcome of a process [12]. Prior to optimization of the efficiency, relatively more sensitive process parameters are identified. In the optimization process, most of the efficiency gain in the pump operation is achieved through controlling these parameters.

The experimental data was collected from the setup shown in Figure 3. The data acquisition module collected and recorded data at 20 points in every second. After getting torque, RPM, flow, and pressure data, efficiency was calculated. Figure 6 illustrates the efficiency of the hydraulic system when a 2 HP pump is used. The efficiency of the system was then calculated.
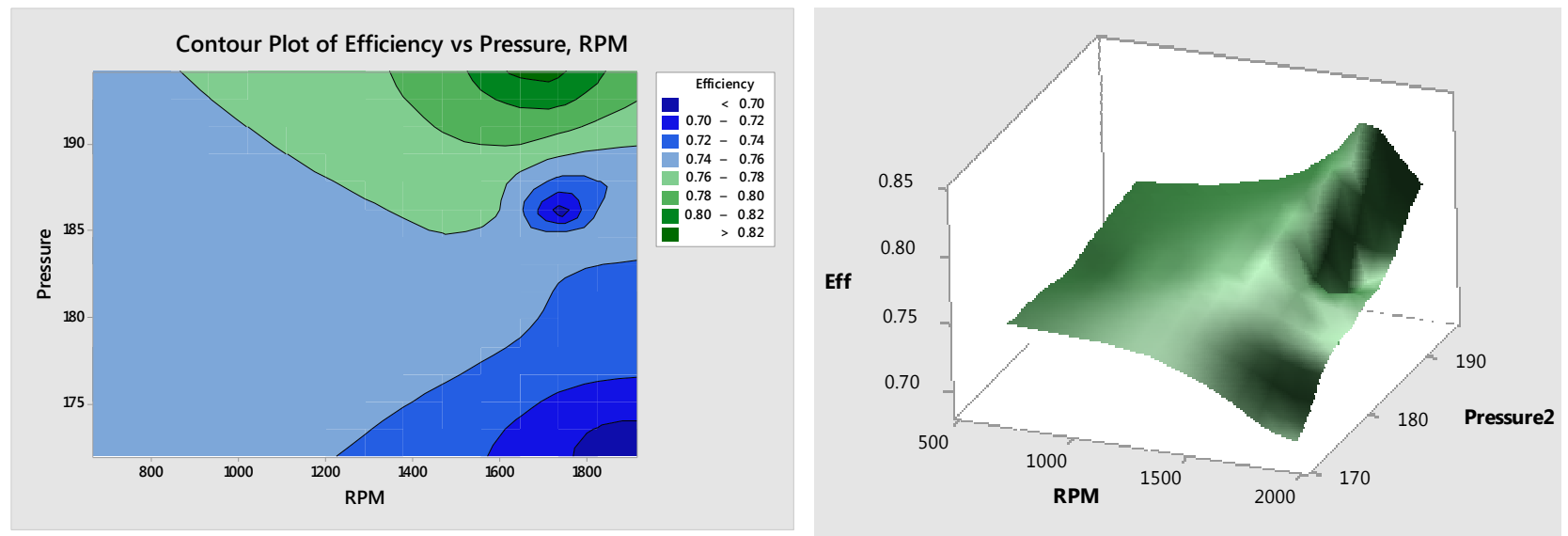

Figure 6: 3D Contour and Surface plot of Efficiency vs Pressure and RPM

At 660 RPM, system's overall efficiency is $74.07 \%$. As RPM increases, the system efficiency also increases. At $1686.36 \mathrm{RPM}$, system efficiency is maximum, $83.14 \%$ which is very close to the known theoretical efficiency of $85.5 \%$ of the pump. After efficiency of the system reaches the highest point, a drop in the efficiency was noticed with increase in RPM.

Upon identifying factors that affect the overall efficiency, the next step is to establish which factors play the most vital role. Determining the best possible settings for these crucial factors that result in the optimum value of overall efficiency is the goal. This analysis represents some observable characteristics of the modeled system. To analyze the existing system, a model was 
created where pressure, temperature and flow in inlet and outlet, RPM and torque were selected as parameters and system efficiency was selected as output. The analysis found that the outlet pressure and RPM were the most significant parameters for optimizing efficiency. Further analysis was done using Excel considering only pressure and RPM. It was found that system gains maximum efficiency (89.34\%) at pump pressure of 196 psi and speed of 1700 RPM.

\section{Conclusion}

A prototype of a hydraulic system is created in the laboratory and the performance data of the pump under all operating conditions within the range of the design limits is acquired. The relationship between the pump's efficiency and operating parameters is established. The data analysis shows that among all operating conditions, the pump pressure and shaft speed effect efficiency the most. A numerical search algorithm is used to identify the optimal operating conditions at the highest efficiency of the pump. At the next level, the effect of other minor parameters will be studied and all operational conditions for the highest efficiency of the pump will be determined. Over time, the efficiency of a system deteriorates due to wear and tear of various components, contamination in fluid, changes in environmental conditions, etc. Real time monitoring of the system as well as determining the optimal operating conditions can contribute to energy saving by operating a system at its highest efficiency in industrial applications.

\section{References}

1. Sullivan, J., Fluid Power Theory and Applications, Prentice Hall Inc., Upper Saddle River, New Jersey, 1998.

2. Rydberg, K.; Energy Efficient Hydraulics - System solutions for loss minimization; National Conference on Fluid Power, Linkoping University, Sweden. March 2015.

3. Choudhury, A. and Rodriguez, J.; Experimental Analysis for Energy-efficient Product Design, Journal of Engineering Technology, Volume 34(1), 2017.

4. Choudhury, A., Rodriguez, P. Ikonomov, J. He, B. De Young, R. Kamm, S. Hinton, Human powered energy efficient vehicle design, Proceedings the American Society for Engineering Education Annual Conference, San Antonio, TX, June 2012.

5. Borghi, M., Zardin, B. Pintore, F., and Belluzi, F.; Energy Savings in the Hydraulic Circuit of Agricultural Tractors, $68^{\text {th }}$ Conference of the Italian Thermal Machines Engineering Association, Energy Proceedia 45, 2014.

6. Kilic, E., Dolen, M., Caliskan, H., Koku, A. B., \& Balkan, T.; Pressure prediction on a variablespeed pump controlled hydraulic system using structured recurrent neural networks. Control Engineering Practice, Vol. 26, pp 51-71, 2014.

7. Puddu, P., \& Paderi, M.; Hydro-pneumatic accumulators for vehicles kinetic energy storage: Influence of gas compressibility and thermal losses on storage capability, Energy, Vol. 57, pp 326-335, 2013.

8. Sola, A., Mota, C., and Kovaleski, J.; A model for improving energy efficiency in industrial motor system using multicriteria analysis. Energy Policy, Vol 39(6), 2011. 
9. Ramakrishnan, R., Hiremath, S., and Singaperumal, M.; Design strategy for improving the energy efficiency in series hydraulic/electric synergy system. Energy, Vol. 67, pp. 422-434, 2014.

10. Kollmar, D., Dr, \& Appel, C. (2010, October 01). Optimising energy efficiency in hydraulic systems. Retrieved January 23, 2018, from

http://www.processonline.com.au/content/pneumatics-hydraulics/article/optimising-energyefficiency-in-hydraulic-systems-1385828778.

11. Choudhury, A. and Rodriguez, J.; A Modular System for Energy Efficiency Study of Hydraulic Applications, Proceedings of the ASEE Annual Conference, New Orleans, LA, June 2016

12. Saltelli A., Ratto M., Andres T., Campolongo F., Cariboni J.,Gatelli D., Saisana M., and Tarantola S.,Global Sensiti-vity Analysis: The Primer, John Wiley and Sons, Ltd., 2008. 